\title{
Supernovae at the Highest Angular Resolution
}

\author{
Schuyler D. Van Dyk \\ IPAC/Caltech, 100-22, Pasadena, CA 91125 USA \\ K.W. Weiler (NRL), R.A. Sramek (NRAO), N. Panagia (STScI), \\ C.K. Lacey (USC), M.J. Montes (NRL), J.M. Marcaide (Valencia), \\ W.H.G. Lewin, D.W. Fox, D. Pooley (MIT), A.V. Filippenko (UC \\ Berkeley), C.Y. Peng (UAriz)
}

\begin{abstract}
The study of supernovae (SNe) and their environments in host galaxies at the highest possible angular resolution in a number of wavelength regimes is providing vital clues to the nature of their progenitor stars. We are observing $\mathrm{SNe}$ in the radio using the Very Large Array (VLA) and VLBI, in the X-rays with Chandra, and in the optical at the subarcsecond resolution of $H S T$.
\end{abstract}

\section{VLA Radio Supernova Observations}

VLA radio supernova observations provide unique information on circumstellar environments and progenitor stellar systems properties. In particular, density changes in the pre-SN stellar wind-established circumstellar matter (CSM) alter the radio emission intensity and allow us to probe CSM structures, and the massloss history, evolution and nature of the SN progenitor star. Recent observations of the Type II SN 1979C in M100 (Montes et al. 2000; cf. Weiler et al. 1991) imply that the SN shock has entered a new, higher-density CSM region. The radio emission has apparently stopped declining and has been constant, or perhaps increasing, from 1990 through 1998, implying that this new region could either be a higher density shell or a "flared" disklike structure. Recent observations of the Type II SN 1980K in NGC 6946 (Montes et al. 1998; cf. Weiler et al. 1992) indicate that the SN has dropped sharply in radio flux density between 1990 and 1994. The ratio $v_{\text {shock }}\left(\sim 10^{4} \mathrm{~km} \mathrm{~s}^{-1}\right)$ to $v_{\text {wind }}\left(\sim 10 \mathrm{~km} \mathrm{~s}^{-1}\right)$ indicates a significant evolution of the relatively massive SN progenitor's mass-loss history $\sim 10^{4}$ years ago, possibly due to a fast "blue-loop" phase.

\section{VLBI Radio Supernova Observations}

Marcaide et al. (1997) mapped with VLBI the expanding shock of the Type IIb SN 1993J in M81. The shell-like radio structure had decelerated 6-42 months after explosion, with $m=0.89$ (where $R_{\text {shell }} \propto t^{m}$ ). In 1996 October, at flux density $S_{6 \mathrm{~cm}}=26 \mathrm{mJy}, R_{\text {shell }}=2.64$ mas $\left(\sim 1.3 \times 10^{17} \mathrm{~cm}\right)$. SN $1979 \mathrm{C}$ 's distance and declining radio power have frustrated previous VLBI detection 
efforts. However, Marcaide et al. (2001, in prep.) successfully detected it in 1999 June at $S_{18 \mathrm{~cm}}=5.4 \mathrm{mJy}$, with all data points in the first lobe. A decade will be needed before the second lobe is sampled, so, the model is not unique: a uniform disk has diameter 4.6 mas $\left(\sim 1.2 \times 10^{18} \mathrm{~cm}\right)$, and a ring, 3.1 mas $\left(\sim 8 \times 10^{17} \mathrm{~cm}\right)$. A $v_{\text {shock }} \sim 8000 \mathrm{~km} \mathrm{~s}^{-1}$ implies $m \simeq 0.62$.

\section{Chandra SN Observations}

The Type IIn SN 1998S in NGC 3877 and Type II-P SN 1999em in NGC 1637 have been detected in Cycle 1 with Chandra. The soft X-ray emission is thermal radiation from the "reverse shock" region of the SN ejecta/dense stellar wind interaction (Chevalier \& Fransson 1994). The unusually low luminosity $\left(L_{X} \approx 2 \times\right.$ $\left.10^{38} \mathrm{erg} \mathrm{s}^{-1}\right)$ from ACIS-S3 observations of SN $1999 \mathrm{em}(7.8 \mathrm{Mpc})$ in 1999 and 2000 (Pooley et al. 2001, in prep.) implies that the progenitor had a low massloss rate. The flux nearly doubled from the third observation to the fourth, and the spectrum continued to soften throughout all observations. Observations of SN 1998S (17 Mpc) in 2000 (Pooley et al. 2001b) yield an unabsorbed $0.2-10 \mathrm{keV}$ luminosity of $L_{X}=1 \times 10^{40} \mathrm{erg} \mathrm{s}^{-1}$. SNe $1998 \mathrm{~S}$ and $1999 \mathrm{em}$ span the extremes of progenitor mass loss.

\section{HST SN Observations}

Examination of the immediate galactic stellar and gaseous SN environments using HST WFPC2 images provide important clues about SN progenitors. For the first time we resolve individual stars in and derive detailed color-magnitude diagrams for several environments, and place rigorous constraints on progenitor masses (Van Dyk et al. 1999a). From detailed photometry of the SN 1979C stellar environment, we find an initial progenitor mass $M \approx 17-18 M_{\odot}$ (Van Dyk et al. 1999b). In addition, we located in a pre-SN archival image the Type IIn SN 1997bs progenitor in M66. Combining ground-based and HST light curves, we conclude that SN $1997 \mathrm{bs}$ is more likely the super-outburst of a very massive $\eta$ Carinae-like luminous blue variable star, rather than a genuine SN (Van Dyk et al. 2000).

\section{References}

Chevalier, R.A., \& Fransson, C. 1994, ApJ, 420, 268

Marcaide, J.M., et al. 1997, ApJ, 486, L31

Montes, M.J., et al. 1998, ApJ, 506, 874

Montes, M.J., et al. 2000, ApJ, 532, 1124

Van Dyk, S.D., et al. 1999a, AJ, 118, 2331

Van Dyk, S.D., et al. 1999b, PASP, 111, 313

Van Dyk, S.D., et al. 2000, PASP, 112, 1532

Weiler, K.W., et al. 1991, ApJ, 380, 161

Weiler, K.W., et al. 1992, ApJ, 398, 248 